\title{
DESCRIPTIVE STUDY OF ENTEROCOCCAL SPECIES WITH THEIR ANTIBIOTIC STEWARDSHIP IN TERTIARY CARE HOSPITAL
}

\author{
Ram Murugan Navaneetha Krishnan'1, Senthil Kumar Jayakumar Jayaraj²
}

${ }_{1}^{1}$ Associate Professor, Department of Microbiology, Government Sivagangai Medical College, Sivagangai, Tamilnadu, India. ${ }^{2}$ Senior Assistant Professor, Department of Microbiology, Government Sivagangai Medical College, Sivagangai, Tamilnadu, India.

ABSTRACT
BACKGROUND
Objectives of this study were
1. Isolation and speciation of enterococci from urine, pus sample, blood and other body fluids.
2. To identify the species of enterococci mostly isolated in ICU and non-ICU samples.
3. To know antibiotic susceptibility pattern of the various isolates.

\section{MATERIALS AND METHODS}

1. Samples such as blood, urine, pus, respiratory samples were collected from patients attending as outpatients and also from different clinical department wards and intensive care units by observing standard specimen collection procedure and aseptic conditions.

2. Identification of Enterococcus species is based on routine bacteriological culture methods and biochemical tests.

3. Antibiotic susceptibility testing was performed with Ampicillin, Penicillin, Levofloxacin, Nitrofurantoin, HL Gentamycin, HL Streptomycin and Teicoplanin with the disc diffusion method (modified Kirby-Bauer method) using Mueller-Hinton agar.

4. Vancomycin Sensitivity test was done with Enterococcus species by E Test Strip using Mueller-Hinton agar. MIC determination paper strip which is coated with Vancomycin in a concentration gradient manner, capable of showing MICs in the range of $0.016 \mathrm{mcg} / \mathrm{ml}$ to $256 \mathrm{mcg} / \mathrm{ml}$, on testing against the Enterococcus organism.

\section{RESULTS}

1. Out of 97 samples isolated, $42 \%$ were from Enterococcus faecalis, $41 \%$ were from Enterococcus faecium, $3 \%$ were from Enterococcus avium, 1\% from Enterococcus gallinarum and 11\% were from other Enterococcus Species.

2. Enterococcus faecalis showed high sensitivity to Ampicillin, Penicillin, Levofloxacin, Nitrofurantoin, Vancomycin and Teicoplanin. Enterococcus faecium and all other species showed high sensitivity to Vancomycin and Teicoplanin only.

3. Enterococcus faecalis were most commonly isolated in ICU and Surgery post-operative ward (IP). Enterococcus faecium were most commonly isolated in OPD, Medical-, Paediatrics- and OG-Wards (IP).

4. Vancomycin and Teicoplanin were most commonly sensitive in OP, Medicine, Paediatrics, \& O\&G Wards. Ampicillin, Penicillin and Levofloxacin, were most commonly sensitive in Surgery\& ICU Wards.

\section{CONCLUSION}

Infection control measures like hand washing, ideal disinfectant usage, and strict antibiotic policy reduces Vancomycin resistance to zero Level in our tertiary care hospital. Enterococcal faecium slowly increasing with most of the antibiotics should be continuously monitored. ICU and surgical ward need more attention to bring down the bugs with antibiotic resistance.

\section{KEY WORDS}

Enterococcus Faecalis, Enterococcus Faecium, Vancomycin.

HOW TO CITE THIS ARTICLE: Krishnan RMN, Jayaraj SKJ. Descriptive study of enterococcal species with their antibiotic stewardship in tertiary care hospital. J. Evolution Med. Dent. Sci. 2019;8(01):37-41, DOI: 10.14260/jemds/2019/9

\section{BACKGROUND}

Enterococci are Gram-positive cocci that often occur in pairs (diplococci) or short chains, and are difficult to distinguish from streptococci based on physical characteristics. Two species are common commensal organisms in the intestines of humans, E. faecalis (90-95\%) and E. faecium (5-10\%). Rare cases of infections occur with other species, including E. casseliflavus, E. gallinarum, and E. raffinosus. ${ }^{1}$

'Financial or Other Competing Interest': None.

Submission 29-11-2018, Peer Review 25-12-2018,

Acceptance 31-12-2018, Published 07-01-2019.

Corresponding Author:

Senthil Kumar Jayakumar Jayaraj,

ED Vairam Kamalam Apartments,

Sambakulam, Madurai-7,

Tamilnadu, India.

E-mail:dr_jsenthil@yahoo.com

DOI: $10.14260 /$ jemds $/ 2019 / 9$

\section{(c) (i) $\odot$}

These organisms become a prominent cause of Nosocomial infection. E. faecalis is most commonly isolated, but incidence of E. faecium is on the rise in many hospitals. ${ }^{1}$ Enterococci are tolerant to extreme temperature $\left(10-45^{\circ} \mathrm{C}\right)$, $\mathrm{pH}$ (4.5-10.0), and high sodium chloride concentrations. ${ }^{2}$ Enterococci exhibit alpha-haemolysis on sheep's blood agar. ${ }^{3}$ Members of the genus Enterococcus were classified as group D Streptococcus with genomic DNA analysis. ${ }^{4}$ Clinical infections caused by Enterococcus include urinary tract infections, bacteraemia, bacterial endocarditis, diverticulitis, Wounds and meningitis. ${ }^{2,3}$ Sensitive strains of these bacteria can be treated with ampicillin, penicillin, Levofloxacin, Teicoplanin, High level Gentamycin, High level Streptomycin, Nitrofurantoin and vancomycin. ${ }^{5}$ Some enterococci are intrinsically resistant to $\beta$-lactam-based antibiotics (Penicillins, cephalosporins, carbapenems), as well as many aminoglycosides. This genus acquires and disseminates resistance to Vancomycin. This is emerging as a nosocomial 
infection of hospitalized patients It produces a serious public health concern.6 VRE may be treated with quinupristin/dalfopristin with response rates around $70 \%$. Tigecycline has also been shown to have antienterococcal activity. ${ }^{7}$ Enterococcus faecium is a leading cause of multidrug resistant enterococcal infections over Enterococcus faecalis. $40 \%$ of medical intensive care units reportedly with E. faecium. Central lines, urinary drainage catheters, and ventilators were related to vancomycin- and ampicillinresistant E. faecium. ${ }^{8}$ The rapid increase of VRE has made it difficult for physicians to fight infections caused by $\mathrm{E}$. faecium. A genome-wide sRNA study suggested that some sRNAs are linked to the antibiotic resistance and stress response in E. faecium. ${ }^{9}$ E. faecalis causes cytolysin in combination with high-level gentamicin resistance is associated with a five-fold increase in risk of death in human bacteraemia patients. 10

\section{MATERIALS AND METHODS}

This study was conducted after getting the ethical committee clearance.

\section{Sample Size}

97

\section{Study Period}

Six months (March 2018 to September 2018)

\section{Inclusion Criteria}

Newly admitted patients without any antibiotic treatment.

\section{Exclusion Criteria}

Patients already with antibiotic treatments, HIV Patients.

This descriptive study was conducted by Department of Microbiology. Government Sivagangai Medical College. Samples were collected from Sivagangai Medical College Hospital. Clinical data were descriptively collected by us using a standardized case-report form. The data that were routinely collected included the patient's age, sex, locations namely ICU, non-ICU ward and outpatient patients with clinical History. The identification of Enterococcal species and antimicrobial susceptibility patterns from 2900 Patients samples. Total number of Enterococci isolates processed was 97. Samples such as Blood, Urine, Pus, Respiratory samples were collected from patients attending as outpatients and also from different clinical departments, wards and intensive care units by observing standard specimen collection procedure and aseptic conditions. The samples were transported to the lab and streaked on MacConkey Agar and Blood Agar medium. After the incubation period at $37^{\circ} \mathrm{C}$, the culture plates were examined. On MacConkey Agar medium, they produce tiny deep pink colonies. They are Heat resistant, surviving at $60^{\circ} \mathrm{C}$ for 30 minutes. On Blood Agar medium, they are non-hemolytic, some strains may show alpha or beta colonies. On selective media, Bile esculin agar slant produces black color colonies. On Gram staining Gram positive cocci appeared as pairs of oval cocci. Catalase test was performed, it was Negative. Motility test was performed with hanging drop method, except E. gallinarum, E. casseliflavus, other species showed non-motile. Identification of Enterococcus species is based on biochemical tests. ${ }^{11}$ E. faecalis was identified with ability to ferment mannitol, Arginine, and
Pyruvate. E. faecium was identified with ability to ferment mannitol, arabinose, arginine. E. avium was identified with ability to ferment mannitol, sorbose, arginine. E. gallinarum was identified with ability to ferment mannitol, raffinose.

Antibiotic Susceptibility testing was performed with Ampicillin, Penicillin, Levofloxacin, Nitrofurantoin, HL Gentamycin, HL Streptomycin and Teicoplanin with the disc diffusion method (Modified Kirby-Bauer method) using Mueller-Hinton agar. ${ }^{12}$ Nitrofurantoin was used for urine samples only. High Level Gentamycin and Streptomycin was used for Blood samples only.

Vancomycin Sensitivity test was done with Enterococcus species by E Test Strip using Mueller-Hinton agar. MIC determination paper strip which is coated with Vancomycin in a concentration gradient manner, capable of showing MICs in the range of $0.016 \mathrm{mcg} / \mathrm{ml}$ to $256 \mathrm{mcg} / \mathrm{ml}$, on testing against the Enterococcus organism. The ellipse intersects the strip in between 2 dilutions, read the MIC as the value which is nearest to the intersection. Vancomycin showing reading of $0.75 \mathrm{mcg} / \mathrm{ml}$ should be rounded up to next concentration i.e. $1.0 \mathrm{mcg} / \mathrm{ml}$. When growth occurs along the entire strip, report the MIC as > the highest values on the MIC strip. When the inhibition ellipse is below the strip (Does not intersect the strip), report the MIC < the lowest value on the MIC scale. MIC of 4 and below reported as sensitive, 8-16 as Intermediate, 32 and above as Resistant. The results were interpreted as per CLSI 2018 (Clinical and Laboratory Standards Institute) guidelines corresponding to that period. All antibiotic discs were obtained from HiMedia (India). ${ }^{13}$

\section{RESULTS}

\begin{tabular}{|c|c|}
\hline Age & Numbers (n=97) \\
\hline Neonates & 6 \\
\hline up to 1 yr. & 3 \\
\hline $1-12$ yrs. & 14 \\
\hline $12-20$ yrs. & 8 \\
\hline $20-40$ yrs. & 18 \\
\hline $40-60$ yrs. & 21 \\
\hline more than 60 yrs. & 27 \\
\hline Total Table 1 \\
\hline \multicolumn{2}{|c|}{} \\
\hline
\end{tabular}

A total of 97 isolates from patients attending the tertiary care teaching hospital. Most common age group of isolation of Enterococci were 20-40 yrs., $40-60$ yrs. and above 60 yrs. [Table. 1]

\begin{tabular}{|c|c|}
\hline Sex & Numbers (n=97) \\
\hline MCH & 12 \\
\hline FCH & 11 \\
\hline Male & 46 \\
\hline Female & 28 \\
\hline Total & $\mathbf{9 7}$ \\
\hline \multicolumn{2}{|c|}{ Table 2 } \\
\hline
\end{tabular}

Out of 97 isolates isolated, 46 isolates were from male patients and 28 isolates were from female patients. 12 isolates were from male children and 11 isolates were from female children. [Table. 2]

\begin{tabular}{|c|c|}
\hline & Numbers (n=97) \\
\hline OP & 6 \\
\hline IP & 67 \\
\hline ICU & 24 \\
\hline Total & $\mathbf{9 7}$ \\
\hline \multicolumn{2}{|c|}{ Table 3 } \\
\hline
\end{tabular}


Out of 97 isolates isolated, most common area of isolation of Enterococci were from medicine, Surgery, O\& G Wards and ICU (NICU, CCU, PICU, IMCU, and IRCU). [Table. 3]

\begin{tabular}{|c|c|}
\hline Samples & Numbers (n=97) \\
\hline Urine & 57 \\
\hline Blood & 15 \\
\hline PUS & 9 \\
\hline Wound Swab & 12 \\
\hline Other Respiratory Samples & 4 \\
\hline Total & $\mathbf{9 7}$ \\
\hline \multicolumn{2}{|c|}{ Table 4 } \\
\hline
\end{tabular}

Out of 97 isolates isolated, most common Samples isolated from Urine, Blood and Wound Swab. [Table. 4]

\begin{tabular}{|c|c|}
\hline Organism & Numbers (n=97) \\
\hline E. faecalis & 42 \\
\hline E. faecium & 41 \\
\hline E. gallinarum & 1 \\
\hline E. avium & 3 \\
\hline E. species & 10 \\
\hline Total & $\mathbf{9 7}$ \\
\hline \multicolumn{2}{|c|}{ Table 5 } \\
\hline
\end{tabular}

\section{Ent.Faecalis Ent.faecium}

Ent.gallinarum $\square$ Ent.Avium

Ent.Species

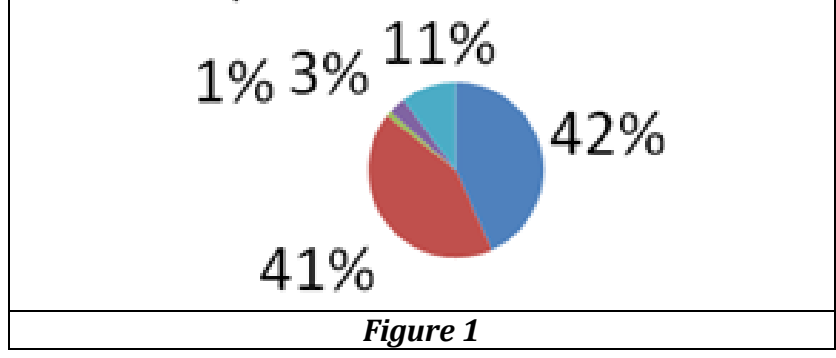

Table 5, Fig. 1, Out of 97 samples isolated, $42 \%$ were from Enterococcus faecalis, $41 \%$ were from Enterococcus faecium, 3\% were from Enterococcus avium, 1\% from Enterococcus gallinarum and $11 \%$ were from Other Enterococcus Species. [Table. 5, Fig. 1]

\begin{tabular}{|c|c|c|c|c|c|c|c|c|c|c|c|c|c|c|c|c|c|c|c|c|c|c|c|c|}
\hline Organisms & \multicolumn{4}{|c|}{ Amp } & \multicolumn{4}{|c|}{ Pen } & \multicolumn{4}{|c|}{ Levo } & \multicolumn{4}{|c|}{ NIT } & \multicolumn{4}{|c|}{ HL GM/HLSM } & \multicolumn{4}{|c|}{ Van/Tei } \\
\hline & $\mathbf{s}$ & $\%$ & $\mathbf{R}$ & $\%$ & $\mathbf{s}$ & $\%$ & $\mathbf{R}$ & $\%$ & $\mathbf{s}$ & $\%$ & $\mathbf{R}$ & $\%$ & $\mathbf{s}$ & $\%$ & $\mathbf{R}$ & $\%$ & $\mathbf{s}$ & $\%$ & $\mathbf{R}$ & $\%$ & $\mathbf{s}$ & $\%$ & $\mathbf{R}$ & $\%$ \\
\hline E. faecalis & 34 & 81 & 8 & 19 & 34 & 81 & 8 & 19 & 31 & 74 & 11 & 26 & 20 & 90 & 2 & 10 & 2 & 50 & 2 & 50 & 42 & 100 & 0 & 0 \\
\hline E. faecium & 4 & 10 & 37 & 90 & 3 & 10 & 38 & 90 & 4 & 10 & 37 & 90 & 7 & 32 & 15 & 68 & 2 & 10 & 9 & 80 & 41 & 100 & 0 & \\
\hline E. species & 5 & 50 & 5 & 50 & 5 & 50 & 5 & 50 & 4 & 40 & 6 & 60 & 6 & 60 & 4 & 40 & 0 & 0 & 0 & & 10 & 100 & 0 & \\
\hline E. avium & 1 & 33 & 2 & 67 & 1 & 33 & 2 & 67 & 2 & 67 & 1 & 33 & 0 & 0 & 0 & 0 & 0 & 0 & 0 & 0 & 3 & 100 & 0 & 0 \\
\hline E. gallinarum & 1 & & 0 & & 1 & & 0 & & 1 & & 0 & & 0 & 0 & 0 & 0 & 0 & 0 & 0 & 0 & 1 & 100 & 0 & 0 \\
\hline
\end{tabular}

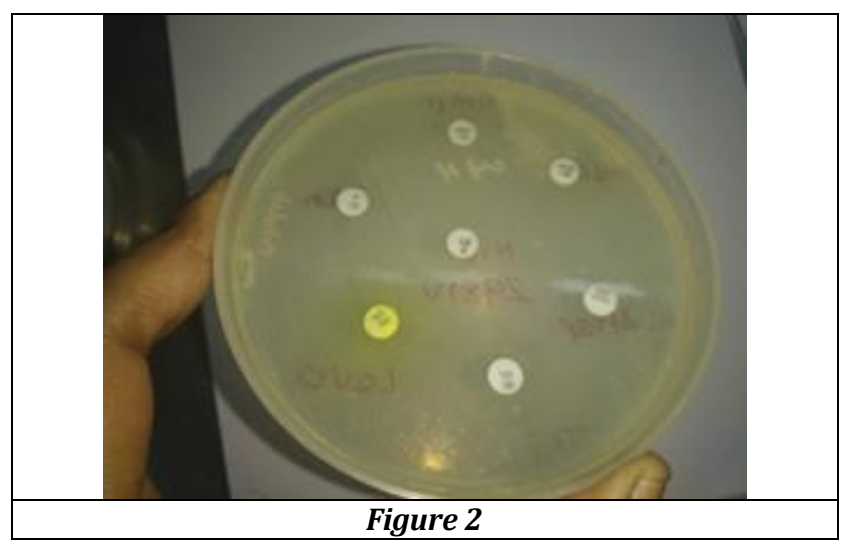

\section{Antibiotic sensitivity analysis for Enterococcus}

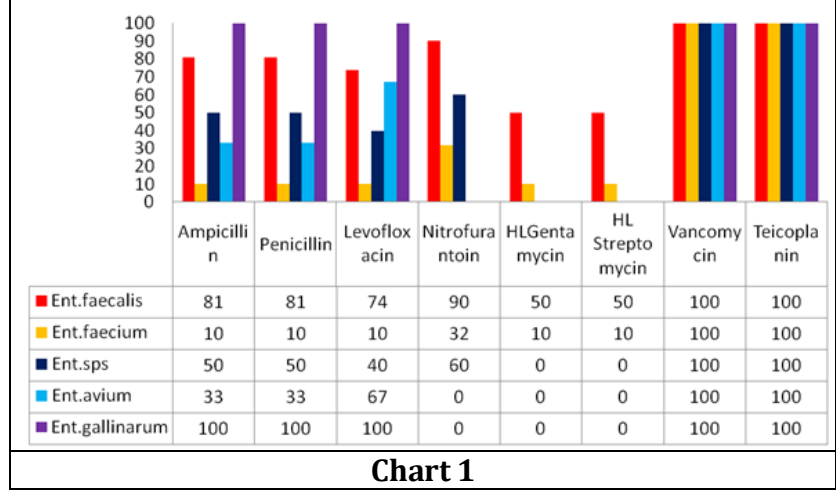

Table 6, Fig. 2, Chart. 1 showed, Enterococcus faecalis showed high sensitivity to Ampicillin, Penicillin, Levofloxacin, Nitrofurantoin, Vancomycin and Teicoplanin. Enterococcus faecium and all other species showed high sensitivity to Vancomycin and Teicoplanin only. [Table. 6]

\begin{tabular}{|c|c|c|c|c|c|}
\hline \multirow[t]{2}{*}{ Sl. No. } & \multirow[t]{2}{*}{ Organisms } & \multicolumn{4}{|c|}{ Vancomycin Mic's with E Strip Method } \\
\hline & & $\begin{array}{l}1 \text { and below } \\
1 \text { in Nos. }\end{array}$ & $\%$ & $\begin{array}{l}\text { Between } \\
1-2 \text { in Nos. }\end{array}$ & $\%$ \\
\hline 1 & E. faecalis & 15 & 36 & 27 & 64 \\
\hline 2 & E. faecium & 36 & 87 & 5 & 13 \\
\hline 3 & E. species & 8 & 80 & 2 & 20 \\
\hline 4 & E. avium & 3 & 100 & 0 & 0 \\
\hline \multirow[t]{2}{*}{5} & E. gallinarum & 1 & 100 & 0 & 0 \\
\hline & Total & 63 & & 34 & \\
\hline \multicolumn{6}{|c|}{ Table 7} \\
\hline
\end{tabular}

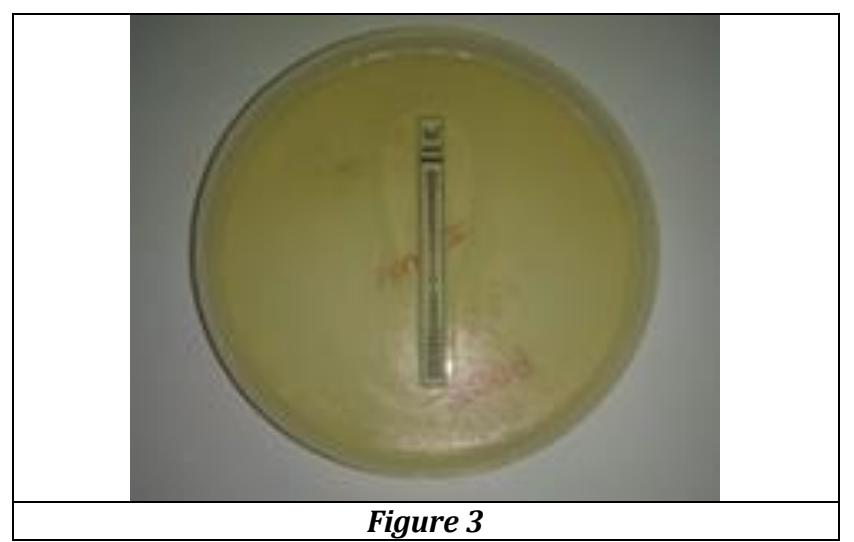


Table 7, Fig. 3 showed, All Species are Sensitive to Vancomycin. Enterococcus faecalis showed $64 \%$ sensitive to MIC between 1 and 2. Enterococcus faecium showed $87 \%$ sensitive to MIC between 1 and below 1. Other Enterococcus species showed $80 \%$ sensitive to MIC between 1 and below 1 .

\begin{tabular}{|c|c|c|c|c|c|c|}
\hline \multicolumn{7}{|c|}{ Most Commonly Isolated Bacteria in Hospital Areas } \\
\hline $\begin{array}{l}\dot{0} \\
z \\
\dot{s}\end{array}$ & 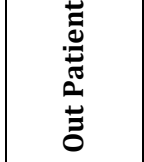 & 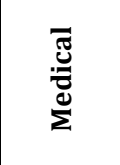 & بّ & 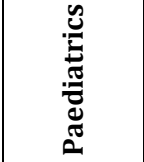 & 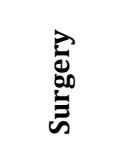 & 己ِ \\
\hline 1 & E. faecium & $\begin{array}{c}\text { E. } \\
\text { faecium }\end{array}$ & $\begin{array}{l}\text { E. faecium } \\
\& \text { E. } \\
\text { faecalis }\end{array}$ & E. faecium & $\begin{array}{c}\text { E. } \\
\text { faecalis }\end{array}$ & $\begin{array}{c}\text { E. } \\
\text { faecalis }\end{array}$ \\
\hline \multicolumn{7}{|c|}{ Table 8} \\
\hline
\end{tabular}

Table 8 showed, Enterococcus faecalis were most commonly isolated in ICU and Surgery Post Operative ward (IP). Enterococcus faecium were most commonly isolated in Op, Medical, Paediatrics and OG Wards (IP). [Table. 8]

\begin{tabular}{|c|c|}
\hline $\begin{array}{c}\text { OP, Medicine, Paediatrics, \& } \\
\text { 0\&G Wards }\end{array}$ & $\begin{array}{c}\text { Surgery } \\
\text { \& ICU }\end{array}$ \\
\hline VAN AND TEICO & AMPI, PENICILLIN, LEVO, \\
& TEICO, VANCO \\
\hline Table 9. Drugs Most Commonly Sensitive in Hospital Area \\
\hline
\end{tabular}

Table 9 showed Vancomycin and Teicoplanin were most commonly sensitive in OP, Medicine, Paediatrics, \& O\&G wards. Ampicillin, Penicillin and Levofloxacin, were most commonly sensitive in Surgery\& ICU Wards.

\section{DISCUSSION}

In this study isolates were highest from urine followed by blood. Most of the studies done on Enterococci support the same findings as Enterococci is identified as one of the most frequent uropathogen. In present study, most common species isolated was $42 \%$ E. faecium and $41 \%$ E. faecalis. In a study by Shweta Jaiswal et al ${ }^{14}$ from north India found also correlates with this study. This may be due to increasing incidence of $\mathrm{E}$. faecium infection in hospitalized patients in present circumstances. Iwen et al. ${ }^{15}$ have also reported an increase in E. faecium isolates from 12.9 to $36.3 \%$ over a period of 8 years during 1987 to 1995.3. Enterococcus has become resistance to many antibiotics due to adhere to indwelling medical devices, and ability to survive in adverse environmental conditions. Antimicrobial resistance in Enterococcus has been increasing mainly in hospitalized patients. 3. In Current study, E. faecalis showed $81 \%$ sensitive to Ampicillin and Penicillin, $74 \%$ sensitive to Levofloxacin, $90 \%$ sensitive to Nitrofurantoin, $100 \%$ sensitive to Vancomycin and Teicoplanin. E. faecium showed higher rates of resistance to Ampicillin, Penicillin, Levofloxacin, Nitrofurantoin. Many studies have also demonstrated that E. faecium is comparatively more resistant than E. faecalis. ${ }^{16} 4$ Zero prevalence of resistance to vancomycin and teicoplanin was detected in both E. faecium and E. faecalis. ${ }^{17}$ In this study, Enterococcus faecalis were most commonly isolated in ICU correlates with Tripathi et al. ${ }^{18}$ Intrinsic risk factors related to the need for intensive care, such as severe underlying disease, multiple illnesses and extremes of age; (2) invasive medical devices, such as endotracheal tubes for mechanical ventilation, intravascular catheters and urinary tract catheters; (3) animate reservoirs (e.g., colonised or infected patients), which increase the risk of cross-infection in the ICU. All Species are Sensitive to Vancomycin. Enterococcus faecalis showed $64 \%$ sensitive to MIC between 1 and 2 . Enterococcus faecium showed $87 \%$ sensitive to MIC between 1 and below 1. Other Enterococcus species showed $80 \%$ sensitive to MIC between 1 and below 1

\section{CONCLUSION}

Infection control measures like hand washing, ideal disinfectant usage, and strict antibiotic policy reduces Vancomycin resistance to Zero Level in our tertiary care Hospital. Enterococcus faecium slowly increasing with most of the antibiotics should be continuously monitored. ICU and surgical wards need more attention to bring down the bugs with antibiotic resistance.

\section{ACKNOWLEDGEMENT}

The author thanks the Dean, Government Sivagangai Medical College, Sivagangai. The author gratefully acknowledges the technicians and other co staff who have helped to conduct this study successfully.

\section{REFERENCES}

[1] Murray BE. The life and times of the Enterococcus. Clinical Microbiology Review 1990;3(1):46-65.

[2] Fisher K, Phillips C. The ecology, epidemiology and virulence of Enterococcus. Microbiology 2009;155(Pt 6):1749-57.

[3] Ryan KJ, Ray CG. Sherri's Medical microbiology. 4th edn. McGraw-Hill 2004: p. 294-5.

[4] Schleifer KH, Kilpper-Balz R. Transfer of Streptococcus faecalis and Streptococcus faecium to the genus Enterococcus nom. rev. as Enterococcus faecalis comb. nov. and Enterococcus faecium comb. nov. Int J Syst Bacteriol 1984;34:31-4.

[5] Pelletier LL Jr. Microbiology of the circulatory system. In: Baron S, edr. Baron's Medical Microbiology. $4^{\text {th }}$ edn. Univ of Texas Medical Branch 1996.

[6] Kurup A, Chlebicki MP, Ling ML, et al. Control of a hospital-wide vancomycin-resistant Enterococci outbreak. American Journal of Infection Control 2008;36(3):206-11.

[7] Tünger A, Aydemir S, Uluer S, et al. In vitro activity of linezolid \& quinupristin/dalfopristin against Grampositive cocci. Indian J Med Res 2004;120(6):546-52.

[8] Higuita A, Huycke MM. Enterococcal disease, epidemiology and implications for treatment. In: Gilmore MS, Clewell DB, Ike Y, eds. Enterococci: from commensals to leading causes of drug resistant infection. Boston, February 2014.

[9] Sinel C, Augagneur Y, Sassi M, et al. Small RNAs in vancomycin-resistant Enterococcus faecium involved in daptomycin response and resistance. Scientific Reports 2017;7(1):11067. 
[10] Huycke MM, Spiegel CA, Gilmore MS. Bacteremia caused by hemolytic, high-level gentamicin-resistant Enterococcus faecalis. Antimicrob Agents Chemother 1991;35(8):1626-34.

[11] Winn W. Konemann's color atlas and diagnostic text of microbiology. $6^{\text {th }}$ edn. Philadelphia, PA: Lippincott Williams \& Wilkins Publishers 2006: p. 945-1021.

[12] Kirby WM, Yoshihara GM, Sundsted KS, et al. Clinical usefulness of a single disc method for antibiotic sensitivity testing. Antibiotics Annu 1956-1957;892-7.

[13] Performance Standards for Antimicrobial Susceptibility Testing. Twenty-Eigth informational supplement. M-100. Clinical Laboratory Standard Institute 2018.

[14] Jaiswal S, Singh A, Verma RK, et al. Characterization, speciation and antimicrobial resistance pattern of Enterococcus species isolated from clinical specimens at a rural tertiary care hospital. International Journal of Research in Medical Sciences 2017;5(8):3484-7.
[15] Iwen PC, Kelly DM, Linder J, et al. Change in prevalence and antibiotic resistance of Enterococcus species isolated from blood cultures over an 8-year period. Antimicrob Agents Chemother 1997;41(2):494-5.

[16] Bhat KG, Paul C, Ananthakrishna NC. Drug resistant enterococci in South Indian hospital. Trop Doct 1998;28(2):106-7.

[17] Arias CA, Reyes J, Zuniga M, et al. Multicentre surveillance of antimicrobial resistance in enterococci and staphylococci from Colombian hospitals, 20012002. J Antimicrob Chemother 2003;51(1):59-68.

[18] Tripathi A, Shukla SK, Singh A, et al. Outcome and risk factor associated with vancomycin-resistant Enterococcus faecalis and Enterococcus faecium at a Tertiary Care Hospital in Northern India. Ind J Med Microbiol 2016;34(1):38-45. 